\title{
Effects of different LED light spectra on growth and immunity of the Japanese eel (Anguilla japonica) and giant mottled eel (A. marmorata)
}

\author{
Yen-Ting Lin ( $\nabla$ b05502093@ntu.edu.tw) \\ National Taiwan University \\ Wei-Chun Hung \\ National Taiwan University \\ Yin-Fu Yeh \\ National Taiwan University \\ Kuang-Mao Lu \\ National Taiwan University \\ Ding-Hwa Cherng \\ National Taiwan University \\ Yu-San Han \\ National Taiwan University
}

\section{Research Article}

Keywords: Anguilla eel, Growth, Immunity, LED, Light spectrum

Posted Date: February 16th, 2022

DOI: https://doi.org/10.21203/rs.3.rs-1233436/v1

License: (1) This work is licensed under a Creative Commons Attribution 4.0 International License.

Read Full License 


\section{Abstract}

Indoor recirculating aquaculture systems make light control possible and enable the usage of specific coloured lights to promote the growth and immunity of aquaculture species. Five different LED wavelengths (white light, red light [622 nm], green light [517 nm], blue light [467 nm], and dark) were used in this study to evaluate growth and immunity in the glass eel stage of two high-valued anguillid species, Japanese eel (Anguilla japonica ) and giant mottled eel ( A. marmorata ). There were no significant differences in growth of the Japanese eel among the groups after 12 weeks of feeding $(P>0.05)$; the survival rate of each group was over $95 \%$. The giant mottled eel showed better growth in total length and body weight in the red light and dark groups $(P<0.05)$. Expression levels of immune-related genes were not significantly different between each group of the Japanese eel and the giant mottled eel $(P>0.05)$. The growth of the Japanese glass eel was not significantly sensitive to different LED wavelengths, while the giant mottled glass eel showed better growth under red light and dark environments. Neither eel species showed significant differences in innate immunity under different LED wavelengths.

\section{Introduction}

Aquaculture has emerged as the most rapidly growing animal food-producing industry in the last two decades (FAO, 2014). Anguillid eels are regarded as an important commercial aquaculture species in East Asia because of their high market demand and nutritional value (Ahn et al., 2015; Shahkar et al., 2015). The Japanese eel Anguilla japonica is a traditionally reared, highly valued anguillid species. However, the amount of natural resources of $A$. japonica available is currently only $5 \%$ that in the 1970 s because of the impact of habitat destruction and overfishing (Chen et al., 2014; Dekker, 2004). According to the International Union for Conservation of Nature and Natural Resources (IUCN), A. japonica has been classified as "Endangered" in the Red list and needs more attention in the eel aquaculture industry (Jacoby \& Gollock, 2014). On the other hand, the cultivation of the giant mottled eel (A. marmorata), which has abundant glass eels and has low fry prices, has increased in Southeast Asia in recent years (Leander et al., 2013; Luo et al., 2013). However, the low growth rate and high mortality rate caused by disease are still considered the biggest obstacles in the development of giant mottled eel aquaculture (Han, 2010).

Indoor recirculating aquaculture systems (RAS) have become increasingly popular because of environmental pollution, lack of water resources and land, and frequent extreme weather events (Martins et al., 2011). Indoor RAS can reduce the water exchange rate to 1/30-1/50 that of traditional outdoor aquaculture ponds, and improve the production capacity by more than ten times (Deviller et al., 2005; Martins et al., 2011). A. japonica and A. marmorata are usually reared in an enclosed RAS at high density (Hsu, 1997; Li, 2018). Fish tend to be more easily affected by disease in intensive aquaculture systems due to the stress-induced weakening of their immune systems (Raman et al., 2013). Aquaculturists have traditionally used antibiotics in rearing systems to control various diseases. The overuse of antibiotics causes many negative side effects, such as drug resistance, decline of the immune system, environmental pollution, and food safety issues (Bachere et al., 2003; Pelgrift et al., 2013; Cabello et al., 
2013). In addition, previous studies have shown that fish in intensive RAS usually show growth retardation and impaired larval development (Davidson et al., 2009; Martins et al., 2009). Therefore, it is imperative to determine an environmentally sustainable method to improve the immunity and growth rate of the cultured species to ensure efficient usage of indoor RAS.

Many studies have indicated that the use of a specific light wavelength (light colour) under different light intensities and photoperiods can improve the growth performance of fish and fish embryos (Boeuf and Le Bail, 1999; Ruchin 2004; Han et al., 2005; Marchesan et al., 2005). For example, the juvenile stage of Candidia barbata tends to have the best body length growth rate under blue light (460-470 nm) and the worst growth rate under red light $(620-630 \mathrm{~nm}$ ) (Chang, 2016). The guppy Poecilia reticulata grows better under blue light, while the Chinese sleeper Perccottus glenii has a higher growth rate under blue and green light. The crucian carp Carassius carassius, the Atlantic halibut Hippoglossus hippoglossus, the rainbow trout Oncorhynchus mykiss, and the silver carp Hypophthalmichthys molitrix showed the best growth under green light and the worst growth under red light (Radenko, 1991; Boeuf \& Pierre-Yves, 1999; Ruchin et al., 2002; Ruchin, 2004; Luchiari \& Pirhonen, 2008). The preleptocephalus stage of the European eel $A$. anguilla showed the best survival rate under low luminosity of red light with a normal photoperiod (Politis et al., 2014). Glass eel-stage A. marmorata individuals with a small amount of pigmentation on the skin tend to stay in areas without light, while those without pigmentation tend to stay under red light environments (Mo et al., 2019). Moreover, light of different colours can also affect the innate immune system of the goldfish Carassius auratus (Eslamloo et al., 2015). Furthermore, Boeuf \& Pierre-Yves (1999) showed that lights of different colours may promote the growth of gonads. For example, the blue damselfish Chrysiptera cyanea showed the best gonad development under red light, while 0 . niloticus showed better gonad development under blue light.

Currently, there are no studies comparing the effect of lights of different colours on the growth and immune response of $A$. japonica or $A$. marmorata. Since RAS is mostly an indoor system, it is easy to control light artificially. Therefore, we aimed to determine the effect of light spectrum on the growth and immune response in the glass eel stages of the Japanese eel and the giant mottled eel cultured in an indoor RAS system.

\section{Materials And Methods}

\subsection{Experimental animals and feeding}

Glass eels of A. japonica and A. marmorata were caught from eastern Taiwan (A. japonica from the Yilan River, $24.7163^{\circ} \mathrm{N}, 121.8348^{\circ} \mathrm{E}$, and A. marmorata from Xiugulan River, $\left.23.4612^{\circ} \mathrm{N}, 121.5008^{\circ} \mathrm{E}\right)$. Eel sampling was approved by the Fishery Agency, Council of Agriculture, Executive Yuan, Taiwan. The specimens were transported at low temperatures through live fish bags filled with oxygen. The health condition of the eels was checked upon arrival at the laboratory located at the Institute of Fisheries Science of National Taiwan University, Taipei. Individuals in good condition were disinfected with $2.5 \mathrm{ppm}$ of potassium permanganate $\left(\mathrm{KMnO}_{4}\right)$ solution for $10 \mathrm{~min}$ to avoid pathogen 
contamination of the experimental system. After sterilization, the eels were kept in five sets of indoor RAS systems with five tanks $(30 \times 30 \times 45 \mathrm{~cm}$ ) for each set and maintained in freshwater for three days before feeding. Photoperiods were set at $12 \mathrm{~h}$ light (7:00-19:00) and $12 \mathrm{~h}$ dark.

The initial body weight and total length of A. japonica and A. marmorata (20 A. japonica for each tank; $30 \mathrm{~A}$. marmorata for each tank in triplicates) were measured before experiment started $(56.7 \pm 2.0 \mathrm{~mm}$, $0.14 \pm 0.01 \mathrm{~g}$ for A. japonica; $51.04 \pm 2.1 \mathrm{~mm}, 0.15 \pm 0.02 \mathrm{~g}$ for A. marmorata). An LED (EVERLIGHT Electronics Co., Ltd., Taiwan) was used as the light source to control the background spectra for the experiment. Each set of RAS included five tanks ( $30 \mathrm{~L}$ water/tank), each exposed to either white light, red light (622 nm), green light (517 nm), or blue light (467 nm) under $100 \mathrm{~lx}$ light intensity with photoperiod 12 hours light and 12 hours dakr, or dark (<5 Ix). Each RAS tank was covered by a black board to avoid any light influence from neighbouring tanks or the environment (Fig. 1). The water temperature and pH were controlled between and , respectively, with a water exchange rate of $20 \mathrm{~L} /$ day for each RAS; oxygen was dissolved to near saturation by aeration. Fish were fed with blood worms (Chironomus dorsalis larvae), which was often used as glass eel feed, at an amount of $10 \%$ of their body weight twice a day for a total of 12 weeks. The remaining feed was removed from the tanks an hour after feeding. The experiment was performed in accordance with the recommendations from the Institutional Animal Care and Use Committee for the care of animals used for experimental or other scientific purposes (approval number 'NTU-110-EL-00009').

\subsection{Sample Collection and Analyses}

The total length (to the nearest $0.1 \mathrm{~mm}$ ) and body weight (to the nearest $1 \mathrm{mg}$ ) were measured every two weeks. The percentage weight gain, condition factor, specific growth rate, and the survival rate in each group were calculated as follows:

\section{Percentage weight gain $(\%)=\frac{\text { Final body weight }(\mathrm{g}) \text {-Initial body weight }(\mathrm{g})}{\text { Initial body weight }} \times 100$}

Condition factor $(\mathrm{K})=1000 \times \frac{\text { Body weight }(\mathrm{g})}{\text { Body length }^{\mathbf{3}}(\mathrm{cm})}$

Specific growth rate $=\frac{\ln (\text { Final body weight })-\ln (\text { Initial body weight })}{84} \times 100$

Survival rate $(\%)=\frac{\text { Final } \mathrm{n}}{\text { Initial } \mathrm{n}} \times 100$

Three fish from each tank were randomly selected and sacrificed to obtain head kidney tissues. Three head kidney tissues from the same tank were pooled together and stored in an RNA protecting reagent at -80 before extracting total RNA using an RNA kit (Bioman Scientific Co. Ltd., Taiwan) for next generation sequencing (NGS). Three other eels from each tank were randomly selected and sacrificed to obtain the head kidney, and pooled together for real-time PCR of immune-related genes.

\subsection{NGS}


The whole genome of A. japonica was successfully assembled in our previous study (http://molas.iis.sinica.edu.tw/jpeel/) (Hsu et al., 2015), and was used as a template to annotate the transcriptome data of A. japonica and A. marmorata. The head kidney samples of the white light, red light, green light, and blue light groups were preserved at -80 and subjected to NGS for transcriptome analysis of the head kidney to identify the immune-related genes in each group. The raw RNA-seq data were filtered using the TrimGalore program (Babraham Bioinformatics, Cambridge, UK) to discard adaptors and low-quality reads $(Q<13)$. Low-complexity reads (repeat sequences) were then removed using the PRINSEQ program (ver. 0.20.4). Finally, general read properties were generated using the FastQC program (Babraham Bioinformatics, Cambridge, UK). The MAKER2 (https://www.yandell-lab.org/ software/maker.html) pipeline was chosen for gene prediction and the gene transfer format was generated. The input datasets included RNA-Seq data, PacBio Iso-Seq data, the newly established A. japonica genome, available protein sequences of $A$. japonica, all teleosts from the uniport database, and the pre-existing zebrafish (Danio rerio) gene model. The predicted open reading frames were annotated with homologs in the NCBI nr database (April 2019) using GhostX (Suzuki et al., 2014). Protein sequence features, including signal peptides, transmembrane domains, and domains described in the Pfam database, were detected by SignalP (Petersen et al., 2011), TMHMM (Krogh et al., 2001), and HMMER (3.1b2) (Mistry et al., 2013). Gene Ontology (GO) annotation was performed for genes with detectable Pfam domains according to Pfam2GO. Furthermore, the protein-coding genes were mapped to the canonical pathway database KEGG pathway using the KEGG Automatic Annotation Server (Moriya et al., 2007), setting the parameters for mapping only to the related prokaryotic database in single-directional best hit (SBH) mode; the signal of an innate immune gene can be detected using this website.

The NGS results were further uploaded to the website (http://molas.iis.sinica.edu.tw/jpeel2018/index.php) set up by our laboratory and Academia Sinica for assembly and analysis. This web database is established on the LAMP system architecture (Ubuntu 14.04, Apache 2.04, PHP 5.1 and MySQL 8.0) with the Bootstrap 3 CSS framework (http://getbootstrap.com/), jQuery1.11.1, and jQuery Validation v1.17 to provide an intuitive user experience. The entire system runs in a virtual machine on the cloud infrastructure of the Institute of Information Science, Academia Sinica, Taiwan. The analysis was performed using scripts written in $\mathrm{R}$ (3.4.2). According to the assembly with gene transfer format, raw reads generated from RNA-seq were estimated for the expression profiles via an intuitive graphical interface in Docexpress (https://hub.docker.com/r/lsbnb/docexpress_fastqc) with a built-in process of Hisat2 $\rightarrow$ StringTie $\rightarrow$ Ballgown. The expression level profiles, in the form of fragments per kilobase of transcript per million mapped reads values, were then submitted to Multi-Omics onLine Analysis System (http://molas.iis.sinica.edu.tw/jpeel2018/index.php) for both eel species.

After this analysis, four innate immune-related genes, viz., lysozyme (LZM), superoxide dismutase (SOD), peroxidase (POD), and interleukin-6 (IL-6), which could be detected by NGS in both eels, were selected for real-time PCR (Table 2).

\subsection{Real-time PCR}


Specific candidate genes were selected for real-time PCR based on the results of the NGS analysis. TRIzol reagent (Bioman Scientific Co. Ltd) was used to extract total RNA, and the purity was quantified by spectrophotometry (Medclub Scientific Co. Ltd). Reverse transcription was performed to synthesize complementary DNA (cDNA) for real-time PCR (Bio-Rad). Four immune-related genes, namely, superoxide dismutase (SOD), lysozyme (LZM), peroxidase (POD), and interleukin-6 (IL-6) were selected as the target genes for real-time PCR, and acidic ribosomal protein (ARP) was used as the reference gene. The primers used for real-time PCR are listed in Table 1.

\subsection{Statistical analysis}

All data were analysed by one-way analysis of variance (IBM SPSS Statistics 24.0) to determine the effects of different spectra. Statistical significance was set at $P<0.05$. A significant effect was followed up with the least significant difference test to compare the means.

\section{Results}

\subsection{Growth rate}

The mean initial total length and body weight of the eels from each tank were not significantly different before the start of the experiment (Table 3, Table 4). The growth of A. japonica showed no significant difference in the total length (Fig. 2) and body weight (Fig. 3) among the different groups ( $>0.05)$ after 12 weeks of feeding (Table 3 ). The percentage weight gain, condition factor $(\mathrm{K})$, and survival rate also did not show significant differences among the groups $(P>0.05)($ Table 3$)$.

Although A. marmorata grew much slower than the Japanese eel, its growth rate was significantly different among each of the treatment groups $(P<0.05)$ (Fig. 4; Fig. 5). The mean total length and body weight were significantly higher in the dark and red light groups than in the other groups $(P<0.05)$ (Table 4). The specific growth rate and percentage weight gain in these groups were also significantly higher than those in the green light group. The survival rate was significantly higher in the red light group than in the green light and dark groups. However, there was no significant difference in the condition factor (K) among the groups (Table 4). The fastest growing period of the giant mottled eel occurred from the sixth to the eighth week (Fig. 4; Fig. 5). On the other hand, the white, green, and blue light groups showed some growth retardation during the eighth to the tenth week. The red light group showed no decrease in growth during the entire experimental period.

\subsection{Real-time PCR}

Real-time PCR was conducted for precise quantification to compare whether the innate immunity of both eel species was affected by different light spectra. The target genes of real-time PCR were SOD, LZM, POD, and IL-6, and acidic ribosomal phosphoprotein (ARP) was used as a reference gene. The results for A. japonica indicated that although the dark group showed higher SOD expression, there was no significant difference among the groups $(P>0.05)$ (Fig. 6). The expression levels of LZM in the red light 
and dark treatment groups were higher than in others, but there were no significant differences among groups $(P>0.05)$ (Fig. 7). The white light group showed the highest expression of IL-6; however, there was no significant difference among the groups $(P>0.05)$ (Fig. 8). The expression of POD was highest in the green light group but was not significantly different from the other groups $(P>0.05)$ (Fig. 9).

The real-time PCR results of the giant mottled eel showed that SOD expression was higher in the dark group than in the other groups, but there was no significant difference among the groups $(P>0.05)$ (Fig. 6). The expression level of LZM in white light and red light groups was the highest; however, there was no significant difference among the groups $(P>0.05)$ (Fig. 7). The red light and white light groups also showed higher expression levels of LZM than the other groups, but without a significant difference ( $P$ > 0.05) (Fig. 8). The POD expression levels in the blue light group were lower than those in others; however, there was no significant difference among the groups $(P<0.05)($ Fig. 9). Moreover, comparison of the qPCR results between both eel species showed no significant differences $(P>0.05)$ in the expression levels of the four immune-related genes under all light spectra (Figs. 6-9).

\section{Discussion}

An earlier study has shown that some fish showed different growth rates under a specific background light spectrum, but the most suitable spectrum differed among species. For example, the pikeperch Sander lucioperca exhibits the highest growth rates and cortisol levels under white light and the lowest under blue light due to the enhancement of cone cells for visual sensitivity under longer-wavelength light, thus improving their growth (Luchiari et al., 2009). Others, such as the barramundi Lates calcarifer, show the best growth rate under red light environments but the worst under green light, because the increase in spectral sensitivity under longer wavelength conditions enhances their feeding behaviour (Jeremy et al., 2011). Different spectra may affect fish visual systems and further influence their physiological functions, such as growth, immune response, endocrine system, etc.

There were no significant differences among the groups in the growth experiment of the glass eel stage of the Japanese eel. Interestingly, Japanese eels in the blue light group showed better feeding motivation than the other groups. Red light could stimulate the feeding motivation in Nile tilapia Oreochromis niloticus but did not improve its growth (Volpato et al., 2013). McLean et al. (2018) indicated that tank colour did not affect the growth performance of juvenile flounder or tilapia, although fish maintained in red-light tanks showed better percent increases in body weight and lower plasma cortisol levels. Some studies have also pointed out that rearing under different light spectra may not change the growth rate of juvenile fish but may have different effects on other behaviours (Villamizar et al., 2011). Such behavioural effects in Japanese eels cultured under different spectra need to be studied further.

On the other hand, the giant mottled eel clearly showed significantly better growth in the dark and red light groups $(P<0.05)$. Aquatic creatures use photoreceptor cells with the highest photosensitivity at a specific wavelength ( $\lambda \max$ ) to detect underwater objects. $\lambda$ max can maximize visual acuity, such as in deep-sea fish (Bowmaker, 1990), or maximize visual contrast, such as in fish inhabiting shallow water or 
coastal areas (Lythgoe, 1979). Therefore, fish tend to live in environments with the best spectral conditions (Downing \& Litvak, 2001). Light of specific colours may enhance their growth potential by facilitating food capture or detection (Pérez et al., 2019). In addition, there may be some potential nonvisual effects of light on endocrine secretion in non-mammalian vertebrate brains, such as that of growth hormone or thyroid hormone (Jonathan et al., 2019). The giant mottled eel shows a blue-shifted rod photoreceptor during its upstream migration stage (Wang et al., 2014), which is insensitive to red light. Moreover, eels are nocturnal animals that prefer to stay away from light. Therefore, it is likely that red light or dark surroundings may reduce stress for the giant mottled eel, resulting in better overall growth. The Japanese eel seems to be more insensitive to the environmental spectrum, and this may have resulted in the lack of significant difference in growth among the treatment groups.

Most of the mortality of the two anguillid species in this study, especially that of the giant mottled eel, resulted due to their escape from the tank (Table 3; Table 4). This might be because the eels are less adapted to a specific wavelength, increasing their stress levels and eliciting an escape response. The Japanese eel may be more tolerant to lights of different colour, and is thus well-adapted to the environment, resulting in a high survival rate. The escape rate was generally high for the giant mottled eel, especially in the dark group. However, it also had the largest growth rate, and stress did not seem to be an important factor. Alternatively, an earlier study suggested that the escape behaviour may be a natural instinct for the giant mottled eel (Matsuda et al., 2016), considering that it prefers to migrate in its early life stage.

Interestingly, the body colour of the giant mottled eel in the red light and dark groups was slightly lighter than those of the others, which is similar to the results of an earlier study (Shin \& Choi, 2014). It has been shown that the pigments in fish can respond to the wavelength of the colour of their environmental background (Bayarri et al., 2002). Biofilm attachments were found on the tank wall in some groups in our study. There were attachments with a dark brown muddy biofilm on the bottom of the blue, green, and white light tanks, while the red and dark tanks had no attachments. This may have caused the darker body colour in red and dark groups to adapt the environment without dark attachment.

Lysozyme (LZM) is an important enzyme that shows antiviral, antibacterial, and anti-inflammatory activities (Saurabh \& Sahoo, 2010). LZM also combines and metabolizes advanced glycosylation end products produced from reactive oxygen species that would otherwise accumulate and cause harm to organisms. Similar to the antioxidant system, LZM may also be affected by stress responses (Eslamloo et al., 2015; Zheng et al., 2016; Gao et al., 2017; Li et al., 2018). LZM expression levels increase under a red light environment in the pikeperch Sander lucioperca (Baekelandt et al., 2019). The results of real-time PCR of LZM in both the Japanese eel and giant mottled eel showed no significant difference among groups (Fig. 7), suggesting that different light spectra may not have any significant effect on LZM activity.

SOD and POD are both important components of the antioxidant system, and catalyse the conversion of superoxide into hydrogen peroxide and oxygen to remove reactive oxygen species; they are also key 
components of the Nrf2 pathway (Fattman et al., 2003; Lin et al. 2008; Shao et al., 2010; Li 2012; Liu et al., 2015; Deyashi \& Chakraborty, 2016). The real-time PCR results of both eel species revealed that although the dark group showed the highest SOD value, there were no significant differences among the groups $(P>0.05)$ (Fig. 6; Fig. 9). SOD and POD have been used as biomarkers of stress in previous studies (Abele \& Puntarulo, 2004; Oliva et al., 2012) due to a dramatic change in the mRNA content and activity of SOD and POD in response to stress (Shin et al., 2011; Choi et al., 2016; Osman et al., 2019). The stress level of each eel species in each background spectrum may not have differed significantly from each other in our study.

IL-6 is a chemical secreted by the immune system (Tanaka et al., 2014). It can stimulate the body tissues to activate immune mechanisms, help the growth of cells, promote the activation of immune cells of the acquired immune system, and direct blood cells to help macrophages to destroy the source of infection (Stefan et al., 2017). An increase in its concentration can lead to a cytokine storm (Ana et al., 2020). The results of real-time PCR for both eel species showed no significant differences among the groups (Fig. 8). This suggested that the expressions of the innate immune genes were not affected by different light spectra in either eel species. The results also showed no significant difference in expression levels between both eel species, which indicates that different spectra only affect the growth, but do not change the immune expression in the Japanese eel and the giant mottled eel.

\section{Conclusion}

Growth is not affected by the wavelength of light in Japanese eels. However, it is recommended to avoid rearing giant mottled eels in a short-wavelength environment, and a red light or dark background is more suitable for their growth. Neither of the eel species showed any significant difference in the expression of four innate immune-related genes (LZM, SOD, POD, and IL-6). The regulatory effects of different light spectra on the immune system and endocrine mechanism of fish require further study.

\section{Declarations}

Acknowledgements:

The authors thank the EVERLIGHT Electronics Co., Ltd., Taiwan (09HTB45001), and the Ministry of Science and Technology, Executive Yuan, Taiwan (MOST 109-2313-B-002 -001-MY2) for funding this project. Finally, the authors are thankful to all the reviewers of this manuscript.

\section{Funding}

The authors thank the EVERLIGHT Electronics Co., Ltd., Taiwan (09HTB45001), and the Ministry of Science and Technology, Executive Yuan, Taiwan (MOST 109-2313-B-002 -001-MY2) for funding this project. Finally, the authors are thankful to all the reviewers of this manuscript. 
The authors declare that they have no conflicts of interests.

Ethics approval

The experiment was performed in accordance with the recommendations from the Institutional Animal Care and Use Committee for the care of animals used for experimental or other scientific purposes (approval number 'NTU-110-EL-00009').

Consent to participate

Not applicable.

Consent for publication

Not applicable.

Data Availability

The data that support the findings of this study are available from the corresponding author, Yu-San Han, upon reasonable request.

Code availability

Not applicable.

\section{Author Contributions}

Yen-Ting Lin and Wei-Chun Hung mainly conducted the experiments, analyzed the results, and wrote the manuscript. Yu-San Han designed and supervised the experiments. Yin-Fu Yeh, Kuang-Mao Lu, and DingHwa Cherng supported the funding and supervised the experiments. All authors participated in manuscript writing and interpretation of results. All authors read and approved the final manuscript.

\section{References}

1. Abele, D., \& Puntarulo, S. (2004). Formation of reactive species and induction of antioxidant defence systems in polar and temperate marine invertebrates and fish. Comparative biochemistry and physiology part A: Molecular \& integrative physiology, 138 (4), 405-415.

2. Ahn, J. C., Chong, W. S., Na, J. H., Yun, H. B., Shin, K. J., Lee, K. W., \& P J. K. (2015). An Evaluation of Major Nutrients of Four Farmed Freshwater Eel Species (Anguilla japonica, A. rostrata, A. bicolor pacifica and A. marmorata). Korean Journal of Fisheries and Aquatic Sciences, 48 (1), 44-50.

3. Ana, C., Olivia, S., Andrew, G., Phillips, J., \& Jason, A. T. (2020). The role of IL-6 and other mediators in the cytokine storm associated with SARS-CoV-2 infection. Journal of Allergy and Clinical Immunology, 146(3), 518-534. 
4. Baekelandt, S., Mandiki, S. N., Schmitz, M., \& Kestemont, P. (2019). Influence of the light spectrum on the daily rhythms of stress and humoral innate immune markers in pikeperch Sander lucioperca. Aquaculture, 499, 358-363.

5. Bayarri, M. J., Madrid, J. A., \& Sánchez-Vázquez, F. J. (2002). Influence of light intensity, spectrum and orientation on sea bass plasma and ocular melatonin. Journal of Pineal Research, 32(1), 34-40.

6. Boeuf, G., \& Le Bail, P. Y. (1999). Does light have an influence on fish growth? Aquaculture, 177(1-4), 129-152.

7. Bowmaker, J. K. (1990). Visual pigments of fishes. In Douglas R.\& Djamgoz M. (Eds.), The Visual System of Fish (pp 81-107). London: Chapman and Hall.

8. Carter, C. G., Westbury, H., Crear, B., Simon, C., \& Thomas, C. (2014). Agonistic behaviour in juvenile southern rock lobster, Jasus edwardsii (Decapoda, Palinuridae): implications for developing aquaculture. ZooKeys, 457, 323-337.

9. Chang, C. C. (2016). Study on the influence of the Candidia barbata's growth with the specific wavelength of LED light source. Thesis of Department of mechanical engineering, National Central University.

10. Chen, J. Z., Huang, S. L., \& Han, Y. S. (2014). Impact of long-term habitat loss on the Japanese eel Anguilla japonica. Estuarine, Coastal and Shelf Science, 151, 361-369.

11. Choi, J. Y., Kim, T. H., Choi, Y. J., Kim, N. N., Oh, S. Y., \& Choi, C. Y. (2016). Effects of various LED light spectra on antioxidant and immune response in juvenile rock bream, Oplegnathus fasciatus exposed to bisphenol A. Environmental Toxicology and Pharmacology, 45, 140-149.

12. Fattman, C. L., Schaefer, L. M., \& Oury, T. D. (2003). Extracellular superoxide dismutase in biology and medicine. Free Radical Biology and Medicine, 35, 236-256.

13. Davidson, J., Good, C., Welsh, C., Brazil, B., \& Summerfelt, S. (2009). Heavy metal and waste metabolite accumulation and their potential effect on rainbow trout performance in a replicated water reuse system operated at low or high system flushing rates. Aquacultural Engineering, 41, 136-145

14. Dekker, W. (2004). Slipping through our hand. Population dynamics of the European eel. PhD dissertation. Institute for Biodiversity and Ecosystem Dynamics, University of Amsterdam.

15. Deviller, G., Palluel, O., Aliaume, C., Asanthi, H., Sanchez, W., Franco Nava, M. A., Blancheton, J-P., \& Casellas, C. (2005). Impact assessment of various rearing systems on fish health using multibiomarker response and metal accumulation. Ecotoxicology and Environmental Safety, 61, 89-97.

16. Deyashi, M., Chakraborty, \& S. B. (2016). Pesticide induced oxidative stress and the role of antioxidant defense system in animal body. Harvest, 2, 1-14

17. Jacoby, D., \& Gollock, M. (2014). Anguilla japonica. The IUCN Red List of Threatened Species, 2014, e.T166184A1117791

18. Downing, G., \& Litvak, M. K. (2001). The effect of light intensity and spectrum on the incidence of first feeding by larval haddock. Journal of Fish Biology, 59(6), 1566-1578. 
19. Eslamloo, K., Akhavan, S. R., Eslamifar, A., \& Henry, M. A. (2015). Effects of background colour on growth performance, skin pigmentation, physiological condition and innate immune responses of goldfish, Carassius auratus. Aquaculture Research, 46(1), 202-215.

20. Bachere, E. (2003). Anti-infectious immune effectors in marine invertebrates: potential tools for disease control in larviculture. Aquaculture, 227, 427-438.

21. Cabello, F. C., Godfrey, H. P., Tomova, A., Ivanova, L., Dolz, H., Millanao, A., Buschmann, A. H. (2013). Antimicrobial use in aquaculture re-examined: its relevance to antimicrobial resistance and to animal and human health. Environmental Microbiology, 15, 1917-1942.

22. FAO, (2014). The State of World Fisheries and Aquaculture, Rome, ISBN 978-92- 5-108276-8.

23. Gao, C., Fu, Q., Su, B., Song, H., Zhou, S., Tan, F., \& Li, C. (2017). The involvement of cathepsin F gene (CTSF) in turbot (Scophthalmus maximus L.) mucosal immunity. Fish \& Shellfish Immunology, 66, 270-279.

24. Han, D., Xie, S., Lei, W., Zhu, X., \& Yang, Y. (2005). Effect of light intensity on growth, survival and skin color of juvenile Chinese longsnout catfish (Leiocassis longirostris Günther). Aquaculture, 248(1-4), 299-306.

25. Han, Y. S. (2010). Study of production of potential aquaculture species-Anguilla marmorata. Project report of Council of Agriculture, Executive Yuan.

26. Hsu, H. Y., Chen, S. H., Cha, Y. R., Tsukamoto, K., Lin, C. Y., \& Han, Y. S. (2015). De novo assembly of the whole transcriptome of the wild embryo, preleptocephalus, leptocephalus, and glass eel of Anguilla japonica and deciphering the digestive and absorptive capacities during early development. PloS one, 10(9), e0139105.

27. Jeremy, F. P. U., Tom, G., Nathan, S. H., Andrew, C. B., Richard, P. S., Shaun, P. C., \& Shelby, E. T. (2011). Tank color increases growth, and alters color preference and spectral sensitivity, in barramundi (Lates calcarifer). Aquaculture Volumes 322-323, 235-240

28. Krogh, A., Larsson, B., von Heijne, G., Sonnhammer, E. L. (2001). Predicting transmembrane protein topology with a hidden Markov model: application to complete genomes. Journal of Molecular Biology 305(3), 567-580. doi: 10.1006/jmbi.2000.4315.

29. Leander, N., Tzeng, W. N., Yeh, N. T., Shen, K. N., \& Han, Y. S. (2013). Effects of metamorphosis timing and the larval growth rate on the latitudinal distribution of sympatric freshwater eels, Anguilla japonica and A. marmorata, in the western North Pacific. Zoological Studies, 52:30.

30. Li, B. (2012). Nrf2 signaling pathway and molecular mechanisms regulating its activation. Foreign Medical Science Section of Medgeography, 33, 148-150.

31. Li, L., Ren, W. J., Liu, C. Y., Dong, S. L., \& Zhu, Y. H. (2018). Comparing trace element concentrations in muscle tissue of marbled eel Anguilla marmorata reared in three different aquaculture systems. Aquaculture Environment Interactions, 10, 13-20.

32. Lin, Y. H., Shie, Y. Y., \& Shiau, S. Y. (2008). Dietary copper requirements of juvenile grouper, Epinephelus malabaricus. Aquaculture, 274, 161-165. 
33. Liu, H., He, J., Chi, C., \& Gu, Y. (2015). Identification and analysis of icCu/Zn-SOD, Mn-SOD and ecCu/Zn-SOD in superoxide dismutase multigene family of Pseudosciaena crocea. Fish \& Shellfish Immunology, 43(2), 491-501.

34. Luchiari, A. C., \& Pirhonen, J. (2008). Effects of ambient colour on colour preference and growth of juvenile rainbow trout Oncorhynchus mykiss (Walbaum). Journal of Fish Biology, 72(6), 1504-1514.

35. Luchiari, A. C., de Morais Freire, F. A., Pirhonen, J., \& Koskela, J. (2009). Longer

36. wavelengths of light improved the growth, intake, and feed efficiency of individually reared juvenile pikeperch, Sander lucioperca (L.). Aquaculture Research 40(8), 880-886.

37. Luo, M., Guan, R., Li, Z., \& Jin, H. (2013). The effects of water temperature on the survival, feeding, and growth of the juveniles of Anguilla marmorata and A. bicolor pacifica. Aquaculture, 400-401, 61-64.

38. Lythgoe, J. N. (1979). Ecology of vision. Clarendon Press.

39. Martins, C. I. M., Pistrin, M. G., Ende, S. S. W., Eding, E. H., \& Verreth, J. A. J. (2009). The accumulation of substances in Recirculating Aquaculture Systems (RAS) affects embryonic and larval development in common carp Cyprinus carpio. Aquaculture, 291, 65-73.

40. Marchesan, M., Spoto, M., Verginella, L., \& Ferrero, E. A. (2005). Behavioural effects of artificial light on fish species of commercial interest. Fisheries Research, 73(1-2), 171-185.

41. Martins, C. I. M., Eding, E. H., \& Verreth, J. A. J. (2011). The effect of recirculating aquaculture systems on the concentrations of heavy metals in culture water and tissues of Nile tilapia Oreochromis niloticus. Food Chemistry, 126, 1001-1005.

42. Matsuda, K., Hattori, H., Tomiyama, M., Yada, T., \& Uchida, K. (2016). Environmental conditions on inducing escape behavior and ability of climb a wall in breeding eels. Journal of Fisheries Technology, 8(2), 67-72.

43. McLean, E., Cotter, P., Thain, C., \& King, N. (2008). Tank color impacts performance of cultured fish. Ribarstvo, 66(2), 43-54

44. Mo, W. J., He, Z. J., Yang, Y., Liu, C., Wang, B., \& Wang, J. P. (2019). Phototaxis of Anguilla marmorata based on fish attracting technology of eel passage. Chinese Journal of Applied Ecology, 30(6), 2109-2115.

45. Moriya, Y., Itoh, M., Okuda, S., Yoshizawa, A. C., \& Kanehisa, M. (2007). KAAS: an automatic genome annotation and pathway reconstruction server. Nucleic Acids Research (Web Server issue), W182-5. doi: $10.1093 / \mathrm{nar} / \mathrm{gkm} 321$.

46. Oliva, M., Vicente, J. J., Gravato, C., Guilhermino, L., Galindo-Riaño, \& M. D. (2012). Oxidative stress biomarkers in Senegal sole, Solea senegalensis, to assess the impact of heavy metal pollution in a Huelva estuary (SW Spain): seasonal and spatial variation. Ecotoxicology and Environmental Safety, 75, 151-162.

47. Osman, A. G., Wuertz, S., \& Mohammed-Geba, K. (2019). Lead-induced heat shock protein (HSP70) and metallothionein (MT) gene expression in the embryos of African catfish Clarias gariepinus (Burchell, 1822). Scientific African, 3, e00056. 
48. Pelgrift, R. Y., \& Friedman, A. J. (2013). Nanotechnology as a therapeutic tool to combat microbial resistance. Advanced Drug Delivery Reviews, 65(13), 1803-1815.

49. Pérez, J. H., Tolla, E., Dunn, I. C., Meddle, S. L., \& Stevenson, T. J. (2019). A comparative perspective on extra-retinal photoreception. Trends in Endocrinology \& Metabolism, 30(1), 39-53.

50. Politis, S. N., Butts, I. A., \& Tomkiewicz, J. (2014). Light impacts embryonic and early larval development of the European eel, Anguilla. Journal of Experimental Marine Biology and Ecology, 461, 407-415.

51. Radenko, V. N. (1991). Importance of temperature and light to growth and survival of larval silver carp, Hypophthalmichthys molitrix. Voprosy Ichthyologii 34, 655-663 (in Russian).

52. Raman, R. P., Prakash, C., Marappan, M., \& Pawar, N. A. (2013). Environmental Stress Mediated Diseases of Fish: An Overview. Advances in Fish Research, Vol. V, pp 141-158.

53. Ruchin, A. B., Vechkanov, V. S., \& Kuznetsov, V. A. (2002). Growth and feeding intensity of young carp Cyprinus carpio under different constant and variable monochromatic illuminations. Journal of Ichthyology, 42, 191-199.

54. Ruchin, A. B. (2004). Influence of colored light on growth rate of juveniles of fish. Fish Physiology and Biochemistry, 30(2), 175-178.

55. Saurabh, S., \& Sahoo, P. K. (2008). Lysozyme: an important defence molecule of fish innate immune system. Aquaculture Research, 39(3), 223-239.

56. Shahkar, E., Yun, H., Kim, D. J., Kim, S. K., Lee, B. I., \& Bai, S. C. (2015). Effects of dietary vitamin C levels on tissue ascorbic acid concentration, hematology, non-specific immune response and gonad histology in broodstock Japanese eel, Anguilla japonica. Aquaculture, 438, 115-121.

57. Shao, X. P., Liu, W. B., Xu, W. N., Lu, K. L., Xia, W., \& Jiang, Y. Y. (2010). Effects of dietary copper sources and levels on performance, copper status, plasma antioxidant activities and relative copper bioavailability in Carassius auratusgibelio. Aquaculture, 308, 60-65.

58. Shin, H. S., Lee, J., \& Choi, C. Y. (2011). Effects of LED light spectra on oxidative stress and the protective role of melatonin in relation to the daily rhythm of the yellowtail clownfish, Amphiprion clarkii. Comparative Biochemistry and Physiology Part A: Molecular \& Integrative Physiology, 160(2), 221-228.

59. Shin, H. S., \& Choi, C. Y. (2014). The stimulatory effect of LED light spectra of genes related to photoreceptors and skin pigmentation in goldfish (Carassius auratus). Fish Physiology and Biochemistry, 40(4), 1229-1238.

60. Stefan, R. J., Kevin, W., \& Leonard, C. (2017). The role of IL-6 in host defence against infections: immunobiology and clinical implications. Nature Reviews Rheumatology, 13, 399-409.

61. Suzuki, S., Kakuta, M., Ishida, T., \& Akiyama, Y. (2014). GHOSTX: An Improved Sequence Homology Search Algorithm Using a Query Suffix Array and a Database Suffix Array. PLoS ONE 9(8), e103833. https://doi.org/10.1371/journal.pone.0103833

62. Tanaka, T., Narazaki, M., \& Kishimoto, T. (2014). IL-6 in Inflammation, Immunity, and Disease. Cold Spring Harbor Perspectives in Biology, 6(10), a016295. 
63. Villamizar, N., Blanco-Vives, B., Migaud, H., Davie, A., Carboni, S., \& Sanchez-Vázquez, F. J. (2011). Effects of light during early larval development of some aquacultured teleosts: A review. Aquaculture, 315(1-2), 86-94.

64. Volpato, G. L., Bovi, T. S., de Freitas, R. H., da Silva, D. F., Delicio, H. C., Giaquinto, P. C., \& Barreto, R. E. (2013). Red light stimulates feeding motivation in fish but does not improve growth. PloS One, 8(3), e59134.

65. Wang, F. Y., Fu, W. C., Wang, I. L., Yan, H. Y., \& Wang, T. Y. (2014). The giant mottled eel, Anguilla marmorata, uses blue-shifted rod photoreceptors during upstream migration. PloS One, 9(8), e103953

66. Zheng, J. L., Yuan, S. S., Li, W. Y., \& Wu, C. W. (2016). Positive and negative innate immune responses in zebrafish under light emitting diodes conditions. Fish \& Shellfish Immunology, 56, 382-387.

\section{Tables}

Table 1. Primers used for qPCR amplification.

\begin{tabular}{lll}
\hline Genes & Primer & Sequences \\
\hline ARP (reference gene) & Forward & 5'-GTGCAGCTCATTAAGACCGG-3' \\
\hline & Reverse & 5'-GGCGATATTCCTCACACCCT-3' \\
\hline SOD & Forward & 5'-TAACGTACGACTATGGGGCC-3' \\
\hline \multirow{2}{*}{ LZM } & Reverse & 5'-GCCGCCACCATTAAACTTCA-3' \\
\hline \multirow{2}{*}{ POD } & Forward & 5'-TGCTGGAATGGATGGATACC-3' \\
\hline & Reverse & 5'-GTAATCGCAGTGCTGATGTC-3' \\
\hline IL-6 & Forward & 5'-GACATCACCCGTTTCTGCAA-3' \\
\hline & Reverse & 5'-GTGGATGAAGGAGGGGAACA-3' \\
\hline & Forward & 5'-CCAGATGTCGCTTCACTTCG-3' \\
\hline
\end{tabular}


Table 2. NGS results of $A$. japonica and $A$. marmorata reared in different LED light spectra for 12 weeks

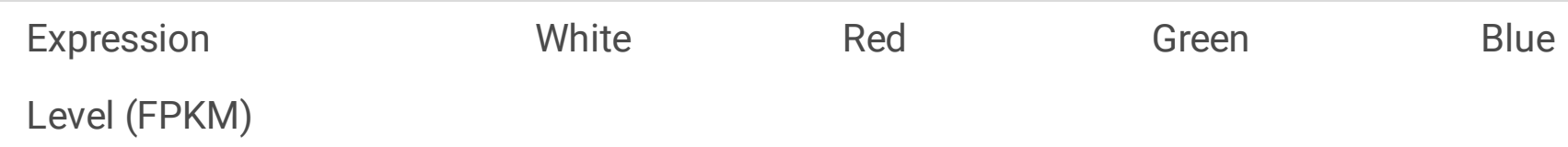

A. japonica

\begin{tabular}{lllll} 
LZM & 54.75 & 159.08 & 85.41 & 134.38 \\
\hline SOD & 257.16 & 282.21 & 349.23 & 269.23 \\
IL-6 & 47.31 & 33.72 & 38.98 & 54.22 \\
POD & 2.42 & 2.52 & 4.90 & 2.27 \\
\hline
\end{tabular}

\begin{abstract}
A. marmorata
\end{abstract}

\begin{tabular}{lllll}
\hline LZM & 5.37 & 5.92 & 0.72 & 2.04 \\
\hline SOD & 493.53 & 297.76 & 410.26 & 342.68 \\
\hline IL-6 & 13.96 & 21.33 & 26.70 & 31.95 \\
POD & 0.34 & 0.14 & 0 & 1.74
\end{tabular}


Table 3. Growth of $A$. japonica reared in different LED light spectra after 12 weeks.

\begin{tabular}{llllll}
\hline & White & Red & Green & Blue & Dark \\
\hline Initial TL (mm) & $56.8 \pm 2.1^{\mathrm{a}^{\mathrm{a}}}$ & $57.0 \pm 2.0^{\mathrm{a}}$ & $56.8 \pm 1.9^{\mathrm{a}}$ & $56.7 \pm 1.6^{\mathrm{a}}$ & $56.3 \pm 2.0^{\mathrm{a}}$ \\
\hline Final TL (mm) & $95.9 \pm 10.2^{\mathrm{a}}$ & $94.0 \pm 10.0^{\mathrm{a}}$ & $93.9 \pm 13.7^{\mathrm{a}}$ & $93.7 \pm 12.5^{\mathrm{a}}$ & $95.7 \pm 7.7^{\mathrm{a}}$ \\
\hline Initial BW (g) & $0.14 \pm 0.01^{\mathrm{a}}$ & $0.15 \pm 0.01^{\mathrm{a}}$ & $0.15 \pm 0.01^{\mathrm{a}}$ & $0.14 \pm 0.01^{\mathrm{a}}$ & $0.14 \pm 0.01^{\mathrm{a}}$ \\
\hline Final BW (g) & $0.78 \pm 0.25^{\mathrm{a}}$ & $0.74 \pm 0.28^{\mathrm{a}}$ & $0.73 \pm 0.22^{\mathrm{a}}$ & $0.75 \pm 0.32^{\mathrm{a}}$ & $0.74 \pm 0.28^{\mathrm{a}}$ \\
\hline SGR (\%) & $1.95 \pm 0.43^{\mathrm{a}}$ & $1.90 \pm 0.66^{\mathrm{a}}$ & $1.88 \pm 0.40^{\mathrm{a}}$ & $1.99 \pm 0.65^{\mathrm{a}}$ & $1.98 \pm 0.65^{\mathrm{a}}$ \\
\hline PWG (\%) & $446.8^{\mathrm{a}}$ & $376.4^{\mathrm{a}}$ & $397.4^{\mathrm{a}}$ & $409.8^{\mathrm{a}}$ & $376.9^{\mathrm{a}}$ \\
\hline Initial number & 40 & 40 & 40 & 40 & 40 \\
\hline Final number & 37 & 39 & 39 & 39 & 39 \\
\hline Survival rate (\%) & $92.5^{\mathrm{a}}$ & $97.5^{\mathrm{a}}$ & $97.5^{\mathrm{a}}$ & $97.5^{\mathrm{a}}$ & $97.5^{\mathrm{a}}$ \\
\hline Initial K & $0.784^{\mathrm{a}}$ & $0.804^{\mathrm{a}}$ & $0.791^{\mathrm{a}}$ & $0.783^{\mathrm{a}}$ & $0.802^{\mathrm{a}}$ \\
\hline Final K & $0.852^{\mathrm{a}}$ & $0.841^{\mathrm{a}}$ & $0.867^{\mathrm{a}}$ & $0.842^{\mathrm{a}}$ & $0.841^{\mathrm{a}}$
\end{tabular}

TL: total length; BW: body weight; SGR: specific growth rate; PWG: percentage weight gain; K: condition factor.

Different letters indicate significant differences between groups $(p<0.05)$. 
Table 4. Growth performance of $A$. marmorata reared in different LED light spectra after 12 weeks.

\begin{tabular}{llllll}
\hline & White & Red & Green & Blue & Dark \\
\hline Initial TL (mm) & $51.0 \pm 2.1^{\mathrm{a}^{*}}$ & $50.7 \pm 2.1^{\mathrm{a}}$ & $51.1 \pm 2.2^{\mathrm{a}}$ & $51.0 \pm 1.6^{\mathrm{a}}$ & $51.4 \pm 2.0^{\mathrm{a}}$ \\
\hline Final TL (mm) & $62.6 \pm 6.1^{\mathrm{a}}$ & $66.0 \pm 6.5^{\mathrm{b}}$ & $63.3 \pm 7.6^{\mathrm{a}}$ & $62.2 \pm 6.1^{\mathrm{a}}$ & $68.0 \pm 7.4^{\mathrm{b}}$ \\
\hline Initial BW (g) & $0.15 \pm 0.03^{\mathrm{a}}$ & $0.15 \pm 0.03^{\mathrm{a}}$ & $0.15 \pm 0.03^{\mathrm{a}}$ & $0.15 \pm 0.02^{\mathrm{a}}$ & $0.15 \pm 0.02^{\mathrm{a}}$ \\
\hline Final BW (g) & $0.32 \pm 0.12^{\mathrm{a}}$ & $0.39 \pm 0.18^{\mathrm{b}}$ & $0.33 \pm 0.13^{\mathrm{a}}$ & $0.31 \pm 0.11^{\mathrm{a}}$ & $0.43 \pm 0.18^{\mathrm{b}}$ \\
\hline SGR (\%) & $0.82 \pm 0.46^{\mathrm{ab}}$ & $1.11 \pm 0.47^{\mathrm{ac}}$ & $0.90 \pm 0.39^{\mathrm{abc}}$ & $0.67 \pm 0.43^{\mathrm{b}}$ & $1.14 \pm 0.38^{\mathrm{c}}$ \\
\hline PWG (\%) & $112.9^{\mathrm{ab}}$ & $173.4^{\mathrm{ac}}$ & $123.7^{\mathrm{abc}}$ & $86.6^{\mathrm{b}}$ & $174.1^{\mathrm{c}}$ \\
\hline Initial n & 90 & 90 & 90 & 90 & 90 \\
\hline Final n & 54 & 68 & 56 & 51 & 39 \\
\hline Survival rate (\%) & $60^{\mathrm{b}}$ & $76^{\mathrm{c}}$ & $62^{\mathrm{b}}$ & $57^{\mathrm{b}}$ & $44^{\mathrm{a}}$ \\
\hline Initial K & $1.106^{\mathrm{a}}$ & $1.080^{\mathrm{a}}$ & $1.093^{\mathrm{a}}$ & $1.120^{\mathrm{a}}$ & $1.078^{\mathrm{a}}$ \\
\hline Final K & $1.245^{\mathrm{a}}$ & $1.262^{\mathrm{a}}$ & $1.182^{\mathrm{a}}$ & $1.206^{\mathrm{a}}$ & $1.384^{\mathrm{a}}$
\end{tabular}

TL: total length; BW: body weight; SGR: specific growth rate; PWG: percentage weight gain; K: condition factor.

Different letters indicate significant differences between groups $(P<0.05)$.

Figures 


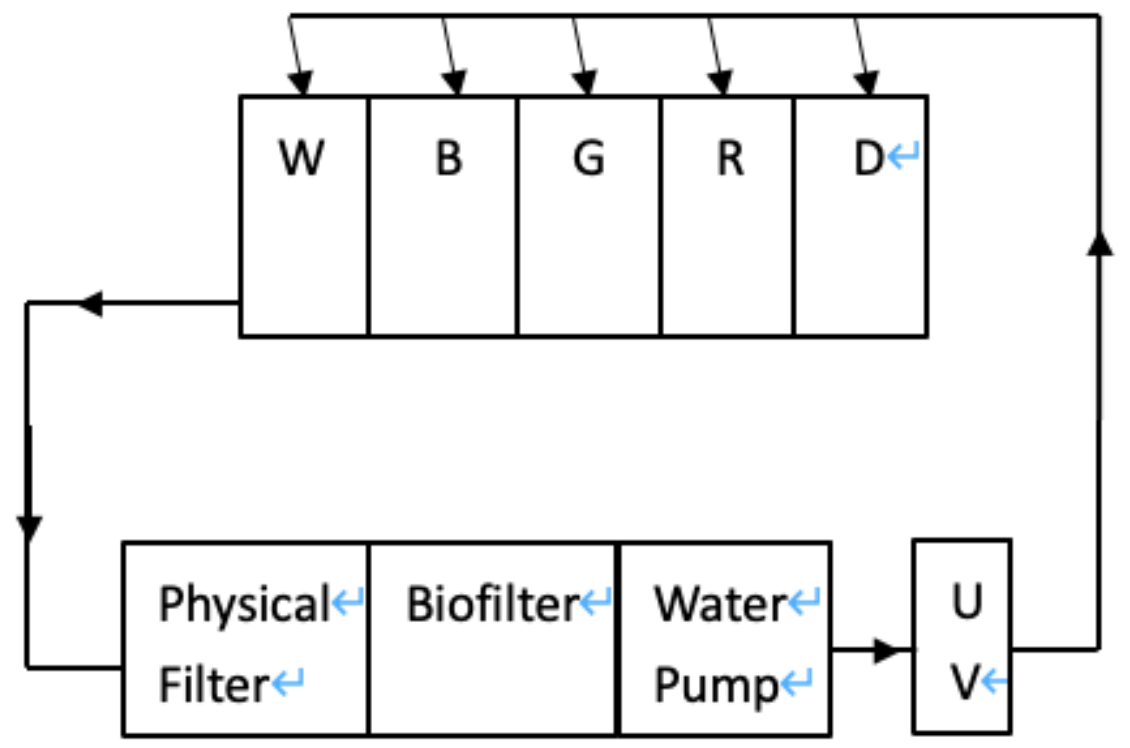

Figure 1

Graph of a set of recirculating aquaculture systems (RAS) used in this study. The five tanks were each 40 $L$ in volume and covered by a black board. W: white light; $R$ : red light (622 nm); G: green light (517 nm); $B$ : blue light $(467 \mathrm{~nm})$.

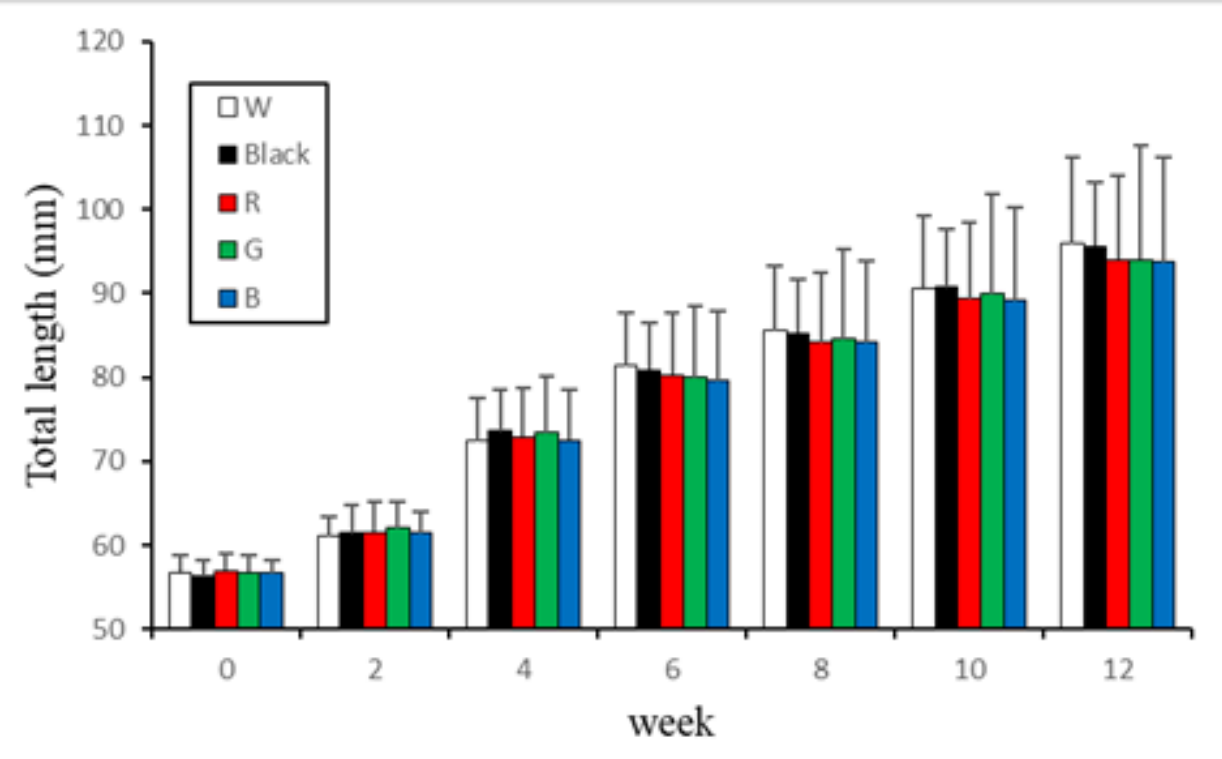

Figure 2 
The total length of Japanese eel reared in different light spectra for 12 weeks. W: white light; Black: dark; B: blue light; G: green light; R: red light

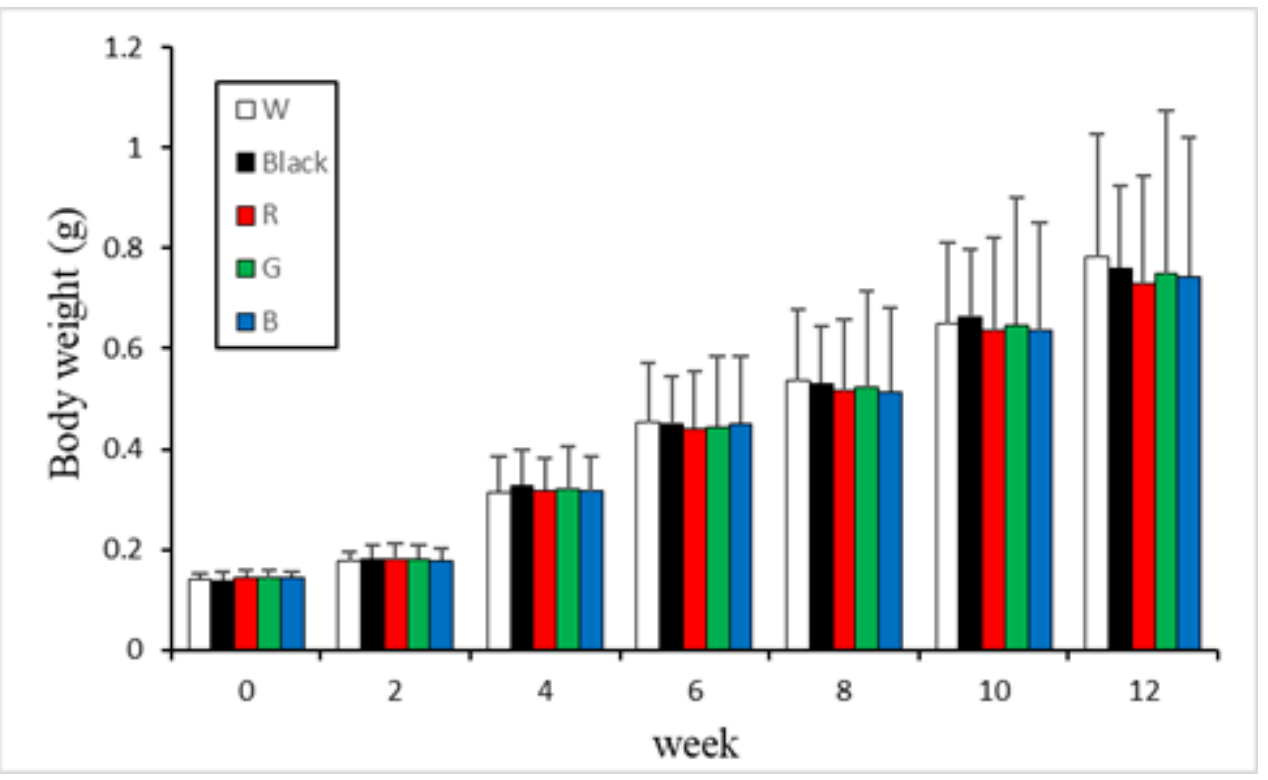

Figure 3

The body weight of Japanese eel reared in different light spectra for 12 weeks. W: white light; Black: dark; B: blue light; G: green light; R: red light

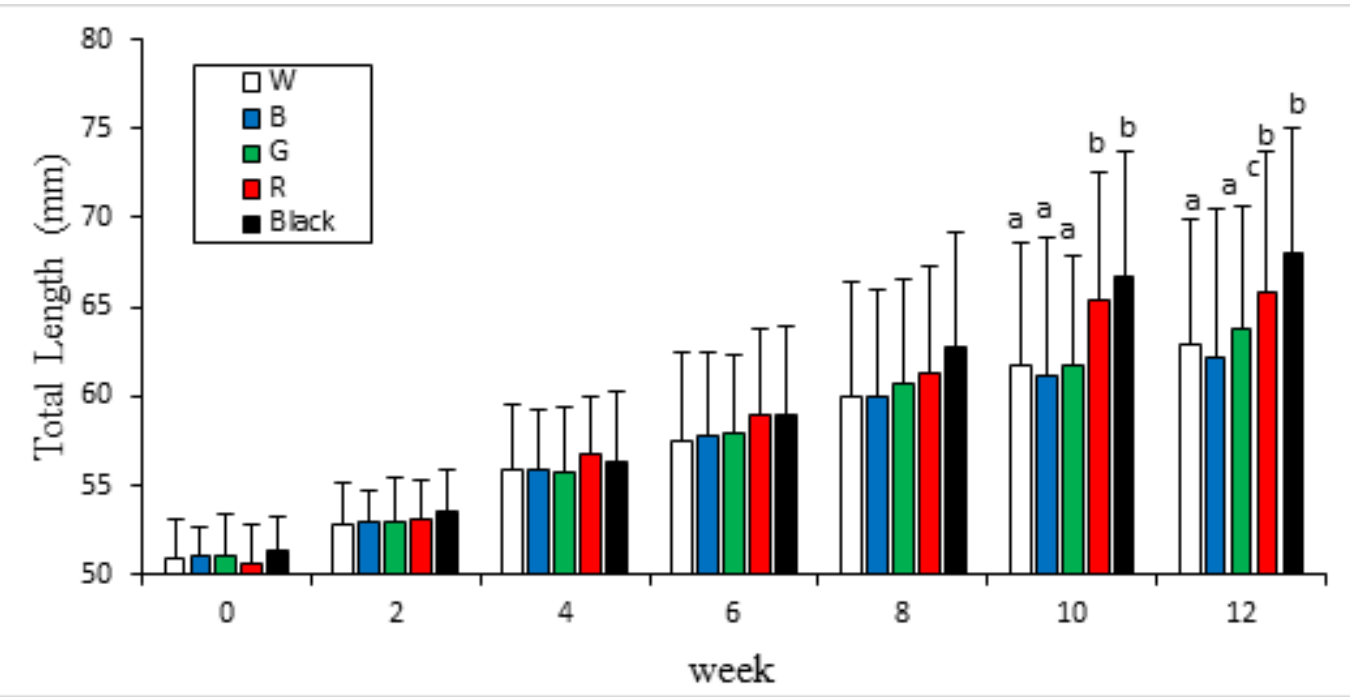

Figure 4

The total length of giant mottled eel reared in different light spectra for 12 weeks. W: white light; Black: dark; B: blue light; G: green light; R: red light. Different letters indicate significant differences between 


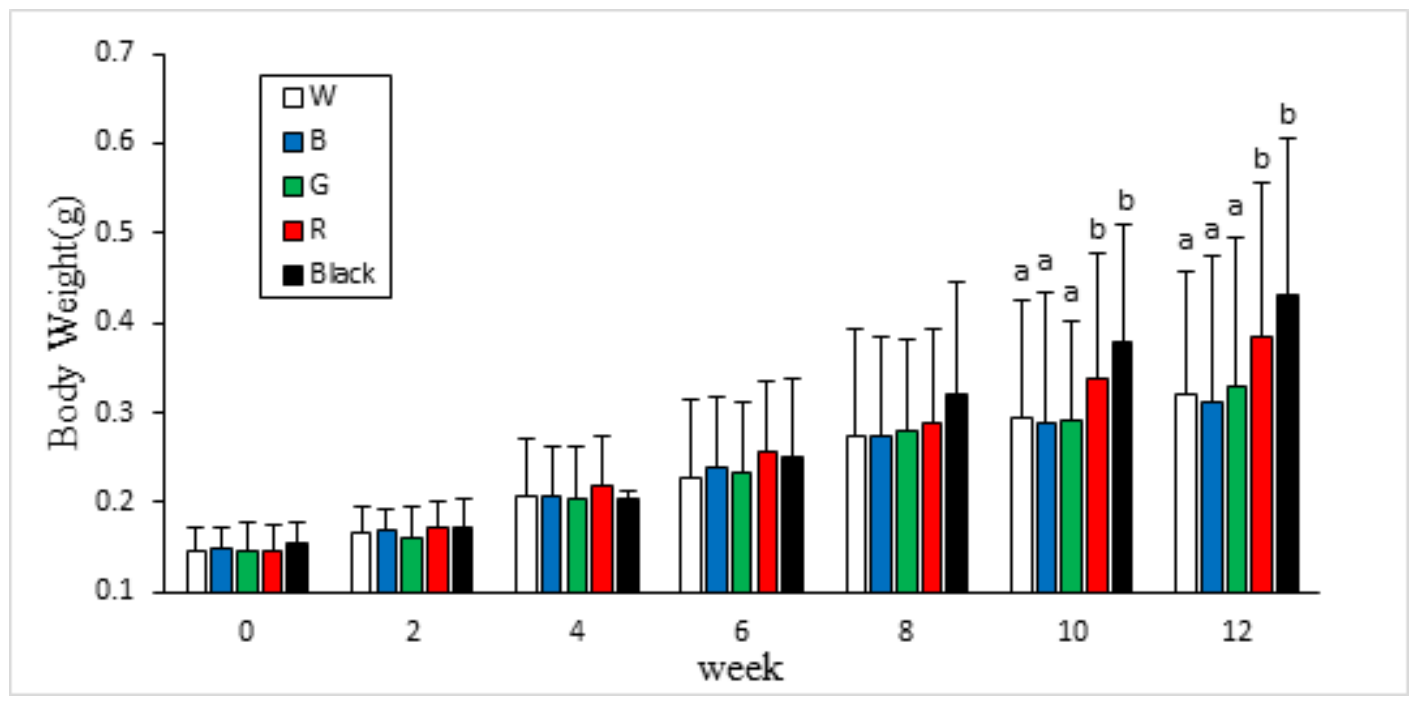

\section{Figure 5}

The body weight of giant mottled eel reared in different light spectra for 12 weeks. W: white light; Black: dark; B: blue light; G: green light; R: red light. Different letters indicate significant differences between groups of the same week $(P<0.05)$.

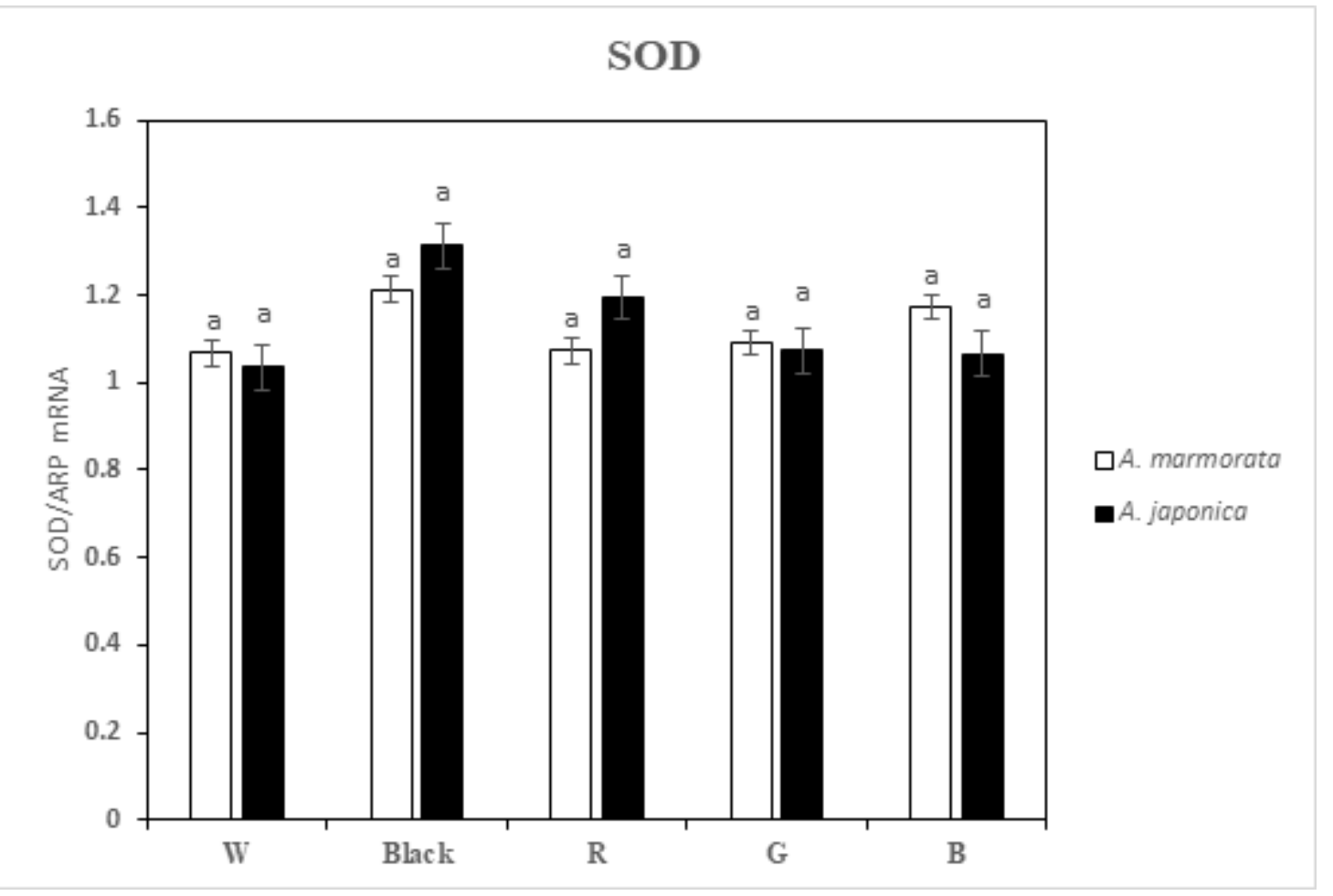


Figure 6

The SOD expression levels of Japanese eel and giant mottled eel reared in different light spectra. W: white light; B: blue light; G: green light; R: red light; black: dark. Different letters indicate significant differences between different spectra groups of the same eel species $(P<0.05)$.

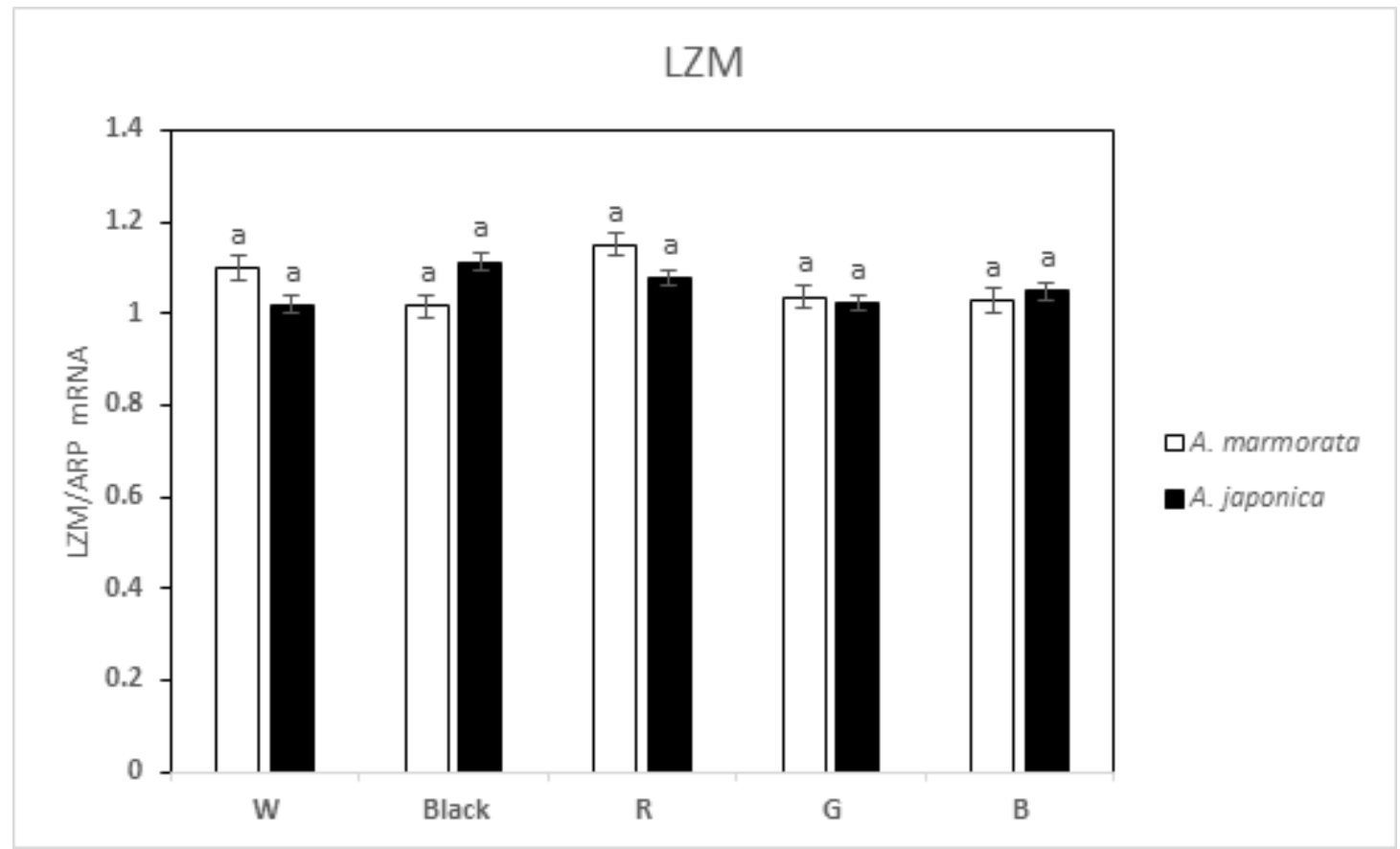

Figure 7

The LZM expression levels of Japanese eel and giant mottled eel reared in different spectra. W: white light; B: blue light; $\mathrm{G}$ : green light; R: red light; black: dark. Different letters indicate significant differences between different spectra groups of the same eel species $(P<0.05)$.

\section{Figure 8}

The IL-6 expression levels of Japanese eel and giant mottled eel reared in different spectra. W: white light; B: blue light; G: green light; R: red light; black: dark. Different letters indicate significant differences between different spectra groups of the same eel species $(P<0.05)$. 


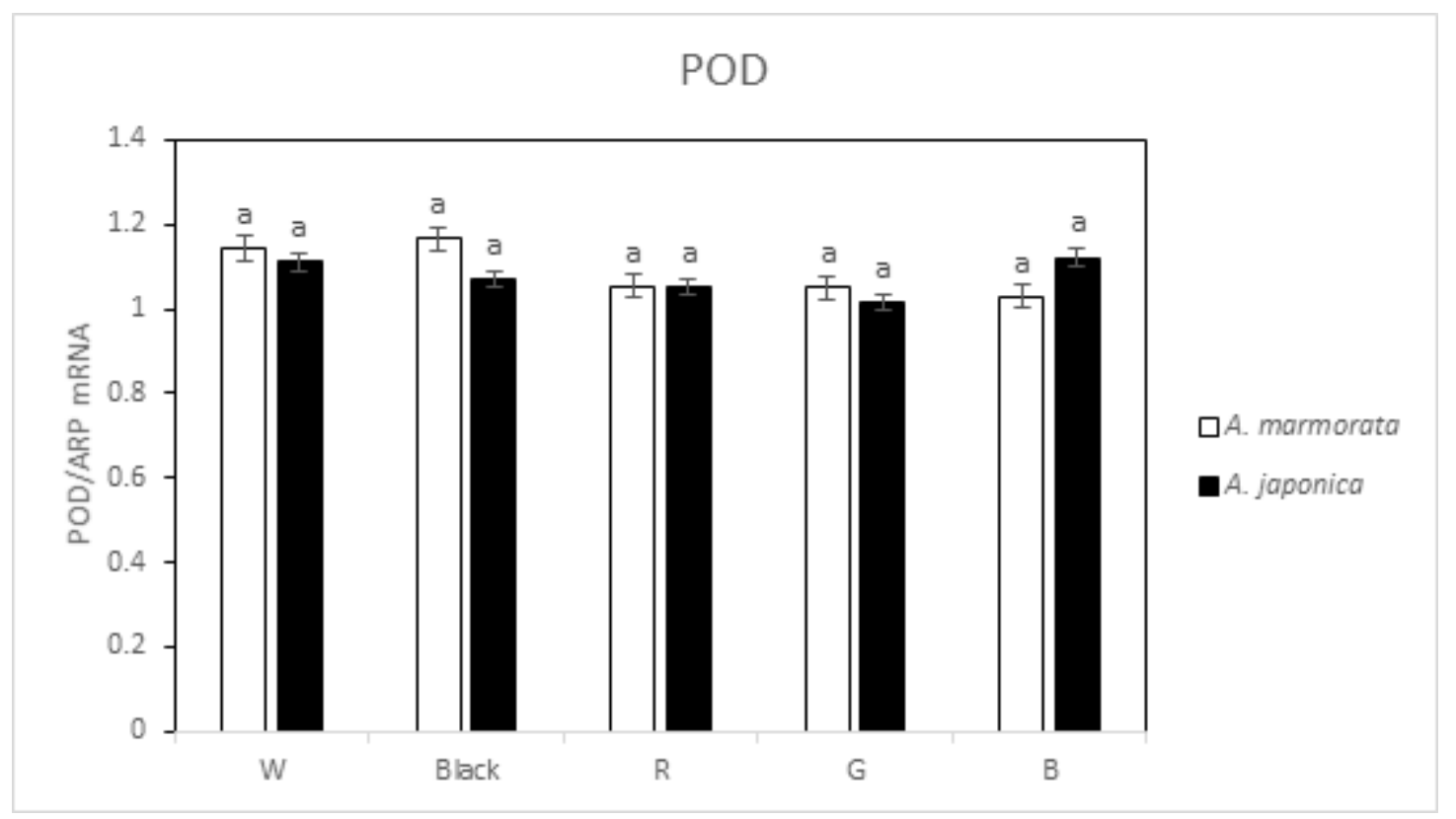

Figure 9

The POD expression levels of Japanese eel and giant mottled eel reared in different spectra. W: white light; B: blue light; G: green light; R: red light; black: dark. Different letters indicate significant differences between different spectra groups of the same eel species $(P<0.05)$. 